\title{
Regards
}

\section{Jean-Marie Legay, homme de réflexion et d'action}

\author{
Jean-Dominique Lebreton \\ Écologue, membre de l'Académie des sciences, CNRS, UMR 5175 CEFE, 34293 Montpellier cedex 5, France
}

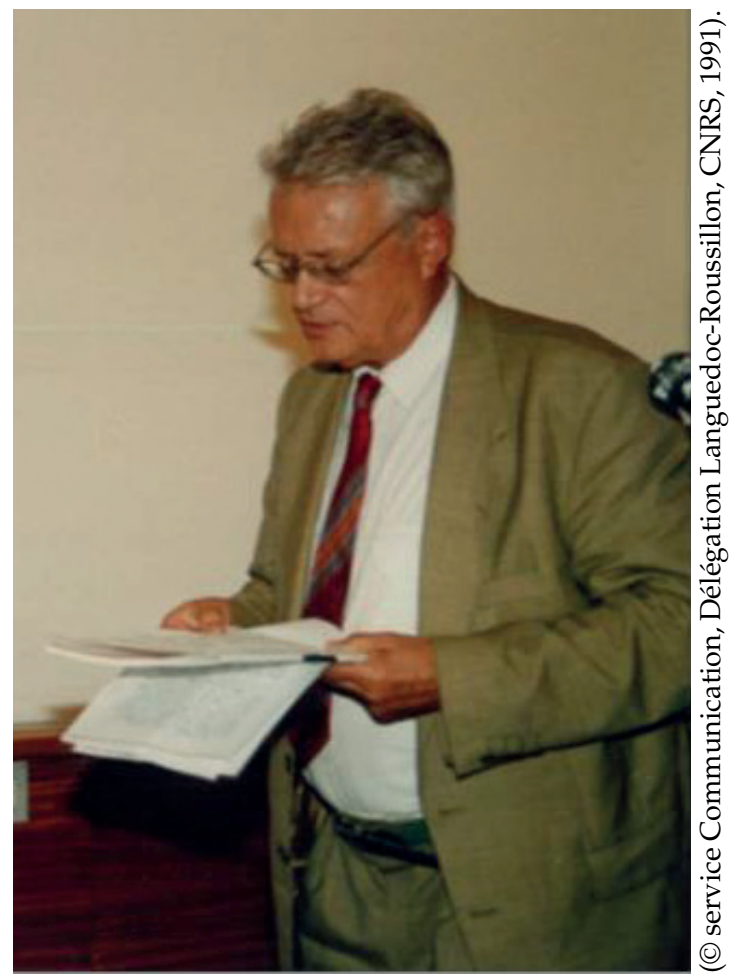

Parler de Jean-Marie Legay est à la fois aisé, tant son influence a été riche et multiforme, et difficile tant un hommage formel lui aurait déplu ${ }^{1}$. Récit d'une rencontre? Chronique de son influence sur mon propre parcours? Regard aussi distancié que possible sur son action ? Privilégier le biomathématicien, le physiologiste des insectes, l'enseignant, le penseur de la modélisation, l'organisateur infatigable, le scientifique curieux de tout, le militant politique?

Auteur correspondant :jean-dominique.lebreton@cefe.cnrs.fr

1 Voir aussi, dans ce numéro et le suivant, les autres hommages à Jean-Marie Legay.
Legay, comme nous l'appelions tous, était un homme de réflexion et d'action au sens le plus fort du terme : s'exprimant rarement autrement que pour agir, n'agissant jamais sans une réflexion et une conviction profonde. Cette rigueur le rendait fort intimidant, cette tension vers l'action souvent déroutant. Cette tension se manifestait de façon paradoxale par une inégalable capacité à patienter face aux obstacles ou à les contourner sans s'encombrer de détails, qu'il s'agisse de calcul matriciel, ce qui ne facilitait pas forcément la tâche des enseignants de travaux dirigés dont j'ai été, ou d'embrouillaminis universitaires, ce qui ne lui a pas toujours valu la bienveillance des collègues. Tous ceux qui l'ont côtoyé se rappellent aussi sa façon inimitable d'écrire son langage parlé qui, jointe à la rigueur de sa pensée, donnait à chacune de ses interventions une hauteur et une force qui, tout en rendant la question ou le commentaire immédiat difficiles, faisaient invariablement naître une réflexion durable.

Changer les mathématiques pour les biologistes, changer la façon de voir les modèles, changer la société, casser le centrage scientifique sur les disciplines..., chacun des engagements de Jean-Marie Legay s'est inscrit dans une durée et s'est construit dans de multiples connexions. Il joignait en effet à une capacité à s'indigner constamment d'injustices ou d'erreurs, une grande ténacité et un énergique refus de l'individualisme, clés de son attitude scientifique comme de son engagement politique.

J'ai ainsi toujours été frappé de l'envergure de son action, au sens de la mise en cohérence d'un puzzle d'activités. Enseignant passionné et passionnant, il a très tôt intégré la notion d'équipe d'enseignement, notion qui fait encore le plus souvent cruellement défaut dans nos universités. Cette capacité à travailler avec les autres, il l'a mise dans les années 1970 au service du groupe de travail "Méthodologie écologique », attaché au comité «Gestion des ressources naturelles renouvelables » de la Délégation générale à la recherche scientifique et technique. Ce groupe a priori obscur, dans des dispositifs 
disparus depuis longtemps, a été un creuset de la pluridisciplinarité autour de l'écologie, traitant aussi bien de la modélisation en écologie que de la gestion de la bilharziose à la Guadeloupe. On peut y voir le socle de bien des actions collectives ultérieures, dont bien sûr NSS.

Passionné par la jeunesse et la vie jusque dans son grand âge, Jean-Marie Legay a toujours gardé un immense respect pour les gens de la terre et pour les gens en difficulté. Que nous laisse-t-il ?

- Une vision de l'écologie qui, au risque d'être anthropocentriste, mais sans réduire le regard à celui de l'agronomie, reconnaît qu'on ne peut traiter longtemps de systèmes écologiques sans se frotter un jour ou l'autre aux sciences de l'homme et de la société.

- Une vision du modèle qui met la modélisation au centre de toute approche de systèmes dynamiques sans être réductionniste, et qui unifie finalement, même si cela n'a pas été une de ses préoccupations primordiales, le modèle statistique et le modèle dynamique.

- Un refus très moderne de concevoir la science comme une vérité préexistante devant être "déversée » à une société en attente.

Le message le plus fort de Jean-Marie Legay me semble être, en effet, de nous faire comprendre que les disciplines scientifiques et les scientifiques eux-mêmes ne se construisent qu' en simplifiant, isolant et compartimentant des questions pour les rendre abordables, et de nous amener à en déduire que le retour vers le monde ne peut se faire sans une patiente reconstruction de la complexité. Bien des scientifiques établis gagneraient à intégrer cette acceptation pleinement réaliste de la complexité du monde et cette modestie. 